\title{
Setting the Table for Change
}

\section{Linda Rosenberg, MSW}

The policy that is made today will shape the health-care practices of tomorrow.

Our community has made unprecedented progress. The passage of the Mental Health Parity and Addictions Equity Act (MHPAEA) and the Patient Protection and Affordable Care Act (ACA) gave us full inclusion in health care. However, as former Centers for Medicare \& Medicaid Services (CMS) Administrator Don Berwick points out, legislation only "sets the table for change." 1

With equality comes entry into the competitive fray of the health-care industry. Legacy healthcare organizations have no more guarantee of success than the mainframe computer companies that were disrupted by personal computers or personal computer companies that were disrupted by tablets and smartphones.

The National Council's policy agenda is filling in the details.

Community behavioral health provider organizations joined voices with military groups, sheriffs' associations, and patients to support Senators Stabenow and Blunt and Representatives Matsui and Lance as they fought for the Excellence in Mental Health Act (the Excellence Act). On March 31, 2014, President Obama signed that legislation into law. ${ }^{2}$

The Excellence Act begins to bring financial parity to the safety net. It creates Certified Community Behavioral Health Clinics that will receive enhanced Medicaid funding to provide comprehensive care via 24/7 evidence-based mental health and addiction services delivered by a skilled workforce operating within a culture of world-class customer service. The Excellence Act rights a wrong and helps community behavioral health providers extend their hands to the men, women, children, and families in their communities. Our charge is to thank Congress for the Excellence Act and lay the groundwork to take it from an eight-state demonstration to a nationwide safety net.

We continue to encourage the Substance Abuse Prevention and Treatment Block Grant to address the fact that a mere $10 \%$ of people with addictions get care. ${ }^{3}$ On our 10th Annual Hill Day, behavioral health advocates asked their members of Congress to add another $\$ 60$ million to this critical component of our nation's safety net.

We also urged the Congress to pass the Behavioral Health Information Technology (IT) Act. Health IT is the bedrock of health care. Technology and the science that underlies it extend our ability to be high touch to providing care anytime, anywhere. When we humanize technology, it allows us to serve the needs of individuals and improve our collective lives. High tech makes high touch possible. We deserve to get the meaningful use of dollars given to others. Without the meaningful use of financial incentives afforded by other health-care sectors, integration is impossible.

Address correspondence to Linda Rosenberg, MSW, National Council for Behavioral Health, Washington, DC, USA. Email: lindar@thenationalcouncil.org.

Journal of Behavioral Health Services \& Research, 2014. 406-407. (c) 2014 National Council for Behavioral Health. DOI 10.1007/s11414-014-9427-6 
With our partners in Maryland and Missouri, we brought Mental Health First Aid to the USA. By the end of this year, a quarter of a million people will have been trained, and through continued advocacy, the Congress appropriated \$15 million to train teachers and school personnel so that our children and grandchildren can have a healthier future. We thank the Congress for this year's \$15 million dollars for the Mental Health First Aid and for an additional \$20 million in 2015.

We know that advocacy works. We can look back on many accomplishments. We know there is still plenty of work to be done. As Dr. Ron Manderscheid recently pointed out, there are still 9.4 million uninsured people currently eligible to enroll in the Medicaid Expansion, and of these, about four million have a prior mental illness or addiction. He notes that those who live in one of the 24 states not currently expanding Medicaid Expansion "will need to engage in vigorous, nontraditional advocacy to alter this tragic situation."4(p.2)

Throughout this special section of the $J B H S \& R$ on the Affordable Care Act, it is clear that behavioral health is critical to the emerging health delivery systems. The articles in this special section of the journal highlight areas and special populations affected by the ACA and other policy changes. Like Manderschied's call to action, Goldman and Karakus outline the populations we will still need to serve throughout public mental health systems. ${ }^{5}$ Andrews highlights the disparities faced by addiction treatment providers in which states cover their services under Medicaid, ${ }^{6}$ and Cuellar discusses the need and opportunity for new models of behavioral health care for the criminal justice population under the ACA. ${ }^{7}$

The laws have been written. The table has been set. Now, it is time for the people who provide the care to improve the care. We are the only ones who can.

\section{References}

1. Berwick D. "The Moral Test." Institute for Healthcare Improvement 23rd Annual National Forum on Quality Improvement in Health Care, Orlando, Florida, December 7, 2011.

2. National Council for Behavioral Health. Excellence in Mental Health Act. Available online at www.thenationalcouncil.org/topics/ excellence-in-mental-health-act/. Accessed 19 June 2014.

3. Open Society Foundations. Defining the Addictions Treatment Gap: Data Summary. Available online at www.opensocietyfoundations.org/ publications/defining-addiction-treatment-gap-data-summary. Accessed 27 June 2014.

4. Manderschied R. Counting the Benefits of the ACA. Available online at www.behavioral.net/blogs/ron-manderscheid/counting-benefits-aca. Accessed 9 June 2014

5. Karakus MC, Goldman HH. Do Not Turn Out the Lights on the Public Mental Health System When the ACA is Fully Implemented. Journal of Behavioral Health Services \& Research 2014; 41(4).

6. Andrews CM. The Relationship of State Medicaid Coverage to Medicaid Acceptance Among Substance Abuse Providers in the United States. Journal of Behavioral Health Services \& Research 2014; 41(4).

7. Cuellar AE, Jehanzeb C. Health Care Reform, Behavioral Health, and the Criminal Justice Population. Journal of Behavioral Health Services \& Research 2014; 41(4). 